Editorial

\title{
Multiple effects of caffeine on physiology
}

\section{Editorial}

Caffeine, a type of methylxanthine, is the most widely used central nervous system stimulant through consumption of coffee, tea, chocolate, and many brands of beverages and energy drinks. The average amount of caffeine consumed in the U.S. population has remained constant at approximately $300 \mathrm{mg}$ per person per day. ${ }^{1}$ On the cellular level, caffeine interferes with multiple signaling pathways. It acts as an antagonist of all four types (A1, A2A, A2B, and A3) of adenosine receptors. It competitively inhibits phosphodiesterases (PDEs). Furthermore, it activates intracellular $\mathrm{Ca}^{2+}$ channel ryanodine receptors (RyRs) to release $\mathrm{Ca}^{2+}$ from the endoplasmic reticulum. ${ }^{2}$ In addition to the psychoactive effects of enhancing alertness and alleviating fatigue, caffeine also has physiological effects on other systems of the body, as seen in a number of recent studies discussed below.

The circadian clock of the body is a primary regulator of the sleep-wake cycle. It is measured by the rhythmic concentrations of the hormone melatonin in the body fluid. A study published on the September 16 issue of Science Translational Medicine by Burke et al. ${ }^{3}$ showed caffeine consumption (200mg, the equivalent of a double espresso) 3hours before bedtime combined with dim-light, compared to dim-light alone, induced a $\sim 40$ min delay of the human circadian rhythm as measured in melatonin secretion in vivo. ${ }^{3}$ They also used human osteosarcoma U2OS cells to study the cellular mechanism since U2OS cells express the key signaling molecules in circadian rhythms such as A1 adenosine receptor (A1R), PDEs, and RyRs. Chronic application of caffeine lengthened the circadian period of molecular oscillations in vitro in U2OS cells. Two other drugs, CGS-15943 and IBMX, were used for their effects on adenosine receptors and PDEs respectively. The authors found caffeine applied effects synergistically with increasing doses of IBMX, but not with CGS-15943, suggesting the action of caffeine on the circadian clock regulation is dependent on adenosine receptors, but not on PDE. Knockdown of the gene ADORAl coding for A1R using siRNA caused an attenuation of caffeine's effect on lengthening the cellular circadian period. This modifying effect of caffeine on the circadian clock may explain the widespread sleep problems and other problems associated with disturbed sleep in populations that heavily consume caffeinated beverages.

Many athletes favour energy drinks containing caffeine in hopes of enhancing endurance as well as short-term, high-intensity physical performance. Early studies have attributed caffeine's performance enhancing effect to reduced pain perception in the central nervous system during exercise. ${ }^{4,5}$ A recent study, published on the May 13 issue of Scientific Reports by Behrens et al. ${ }^{6}$ now linked caffeine to the modulation of muscle strength and activation. ${ }^{6}$ The study showed that caffeine ingestion (oral intake $8 \mathrm{mg} / \mathrm{kg}$ in a gelatin capsule) induced an acute increase of voluntary muscle strength one hour after the intake. This increase of muscle strength was due to the increased voluntary activation of the quadriceps muscle during isometric $(17 \%)$, concentric (17\%) and eccentric contractions (27.6\%), instead of changes of spinal reflex responses.

With or without the taste of coffee, caffeine apparently affects

\author{
Volume 2 Issue 4 - 2015
}

\author{
Ni Song,' He Liu \\ 'Division of Health Sciences \& Workforce Education, Lamar \\ State College-Orange, USA \\ ${ }^{2}$ Department of Biology, Gannon University, USA
}

Correspondence: He Liu, Department of Biology, Gannon University, USA, Email liu0I7@gannon.edu

Ni Song, Division of Health Sciences \& Workforce Technology, Lamar State College-Orange, Orange, USA, Email Ni.Song@Isco.edu

Received: October 19,2015 | Published: October 21, 2015

autonomic arousal, respiratory response, and reported alertness. A study published on the September issue of Psychopharmacology by Benke et al. ${ }^{7}$ studied the subjects' physiology parameters and startle eye blink responses after giving subjects caffeine either with the expectation of arousal induction when mixed in coffee, or without the expectation when mixed in bitter lemon soda. ${ }^{7}$ Regardless if it was offered in coffee or bitter lemon soda, caffeine $(4 \mathrm{mg}$ per $\mathrm{kg}$ body weight) increased skin conductance level, decreased heart rate, increased minute ventilation, decreased end-tidal $\mathrm{CO}_{2}$, and increased reported alertness. Interestingly, the results of behavioural tests in this study suggest both caffeine ingestion and the expectation of arousal (coffee consumption) contribute to the startle eye blink reflex magnitude.

Caffeine, when given in combination with noise, may make hearing impairments even worse. Mujica-Mota et al. ${ }^{8}$ studied the effect of caffeine on hearing impairments following acoustic trauma. ${ }^{8}$ Guinea pigs were exposed to noise $(6 \mathrm{kHz}$ at $120 \mathrm{~dB}$ for one hour) combined with intraperitoneal caffeine $(120 \mathrm{mg} / \mathrm{kg} /$ day for 14 days $)$. These animals showed greater impairment in hearing measured by auditory brainstem responses. In addition, diminished number of spiral ganglion cells was observed together with weight loss and higher alertness. Caffeine did not cause damage in the digestive system, liver, nor the auditory hair cells. This study was published on American Journal of Otolaryngology.

\section{Acknowledgements}

None.

\section{Conflict of interest}

The author declares no conflict of interest.

\section{References}

1. Gonzalez de Mejia E, Ramirez-Mares MV. Impact of caffeine and coffee on our health. Trends Endocrinol Metab. 2014;25(10):489-492.

2. Nehlig A, Daval JL, Debry G. Caffeine and the central nervous system: mechanisms of action, biochemical, metabolic and psychostimulant effects. Brain Res Brain Res Rev. 1992;17(2):139-170. 
3. Burke TM, Markwald RR, McHill AW, Chinoy ED, Snider JA, et al. Effects of caffeine on the human circadian clock in vivo and in vitro. Sci Transl Med. 2015;7(305):305ra146.

4. Motl RW, O’Connor PJ, Dishman RK. Effect of caffeine on perceptions of leg muscle pain during moderate intensity cycling exercise. $J$ Pain. 2003;4(6):316-321.

5. Gliottoni RC1, Motl RW. Effect of caffeine on leg-muscle pain during intense cycling exercise: possible role of anxiety sensitivity. Int $J$ Sport Nutr Exerc Metab. 2008;18(2):103-115.

6. Behrens M, Mau-Moeller A, Weippert M, et al. Caffeine-induced increase in voluntary activation and strength of the quadriceps muscle during isometric, concentric and eccentric contractions. Sci Rep 2015;5:10209.
7. Benke C, Blumenthal TD, Modeß C, et al. Effects of anxiety sensitivity and expectations on the modulation of the startle eyeblink response during a caffeine challenge. Psychopharmacology (Berl). 2015;232(18):34033416.

8. Mujica-Mota MA, Gasbarrino K, Rappaport JM, et al. The effect of caffeine on hearing in a guinea pig model of acoustic trauma. Am J Otolaryngol. 2014;35(2):99-105. 